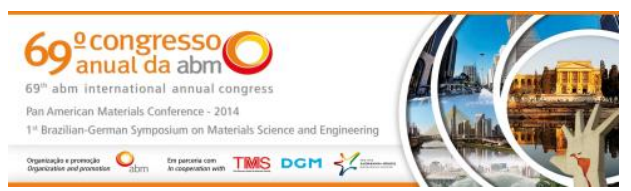

Tema: Materiais cerâmicos, compósitos e poliméricos

\title{
TRATAMENTO QUÍMICO SUPERFICIAL DE FIBRA DE SISAL: MELHORIAS NO DESEMPENHO MECÂNICO DE COMPÓSITOS SEMI-ECOLÓGICOS SISAL/POLIÉSTER ${ }^{\star}$
}

\author{
Jayna Kátia Dionisio dos Santos ${ }^{1}$ \\ Camilla de Medeiros Dantas ${ }^{1}$ \\ Raimundo Carlos Silvério Freire Júnior ${ }^{2}$
}

Resumo

Os materiais compósitos tornaram-se ao longo dos últimos anos, essenciais ao surgimento de novas tecnologias. Para tanto, é imprescindível que alguns cuidados sejam considerados ao desenvolver estes materiais, como o uso do processo de fabricação adequado e a aderência fibra/matriz, sendo este último um fator preponderante na resistência mecânica final do produto. Também se torna cada vez mais necessário o desenvolvimento de materiais com capacidade de utilização ecologicamente correta. Diante disso, foi fabricado um compósito parcialmente ecológico, utilizando como reforço um tecido de sisal, e como matriz resina poliéster. Ainda, buscando a melhoria da aderência fibra/matriz foi efetuado um tratamento com Hidróxido de sódio $(\mathrm{NaOH})$ a uma concentração de $3 \%$ no reforço de sisal. Para a caracterização mecânica do compósito, foram realizados ensaios de tração uniaxial nas fibras tratadas e não tratadas, bem como nas lâminas que utilizaram estas fibras como reforço, mediante a norma ASTM 3039. Quanto a esta propriedade mecânica, foi observado que o tratamento químico superficial diminui expressivamente a resistência à tração da fibra e, em contrapartida, aumentou a resistência do compósito polimérico quando comparado ao que utilizou fibra não tratada, confirmando assim o efeito positivo do tratamento na superfície do reforço de fibra natural.

Palavras-chave: Compósito polimérico; Sisal; Tratamento superficial; Propriedade mecânica.

\section{CHEMICAL SURFACE TREATMENT IN SISAL FIBER: IMPROVEMENTS IN MECHANICAL PERFORMANCE OF COMPOSITE SEMI-ECOLÓGICOS}

\section{Abstract}

I Composite materials have become over the past few years, essential to the emergence of new technologies. For this it is essential that some care be considered when developing these materials, as the use of appropriate manufacturing process and adhesion fiber / matrix, the latter being a major factor in the final product strength. It also becomes increasingly necessary to develop materials capable of Eco-use. Therefore, it was produced a partially eco composite, using a fabric as reinforcement of sisal and polyester resin as matrix. Still, seeking to improve the adhesion fiber / matrix was made a treatment with sodium hydroxide $(\mathrm{NaOH})$ at a concentration of $3 \%$ strengthening of sisal. For the mechanical characterization of the composite tensile tests were performed on treated and untreated fibers, and blades used in these fibers as reinforcement by ASTM 3039. As for this mechanical property, it was observed that the surface chemical treatment significantly decreases the tensile strength of the fiber and, in turn, increased the resistance of the polymer composite compared to that used untreated fiber, thus confirming the positive effect of treatment on the surface strengthening natural fiber.

Keywords: Polymeric composite; Sisal; Surface treatment; Mechanical property.

1 Tecnóloga em Fabricação Mecânica, mestre em engenharia mecânica, doutoranda, Programa de Pósgraduação em Engenharia Mecânica, Universidade Federal do Rio Grande do Norte, Natal, RN, Brasil.

2 Engenheiro mecânico, mestre em engenharia mecânica, doutor em engenharia mecânica, professor doutor, centro de tecnologia, Universidade Federal do Rio Grande do Norte, Natal, RN, Brasil.

\footnotetext{
* Contribuição técnica ao 69ำ Congresso Anual da ABM - Internacional e ao 14ํㅡㄹ ENEMET - Encontro Nacional de Estudantes de Engenharia Metalúrgica, de Materiais e de Minas,21 a 25 de julho de 2014, São Paulo, SP, Brasil.
} 


\section{INTRODUÇÃO}

Os materiais compósitos tornaram-se ao longo dos últimos anos, essenciais ao desenvolvimento de novas tecnologias, principalmente devido às suas características que são únicas, pois são projetados e fabricados para atender as necessidades específicas em cada aplicação, sendo utilizados extensivamente em tecnologias de ponta das mais diversas indústrias como aeroespacial, automobilística, construção civil, petrolífera e de artigos esportivos Santos [1]. Este material é definido por Gay [2] como sendo um material formado por diferentes materiais, sendo que quando analisado macroscopicamente o mesmo é homogêneo, possuindo fibras contínuas ou não, que oferecem resistência mecânica; e, a matriz que dá forma ao produto final.

Também se torna cada vez mais necessário o desenvolvimento de materiais com capacidade de utilização ecologicamente correta, podendo-se utilizar para isso, reforços de fibras naturais em compósitos poliméricos, que tornam o material ecologicamente correto, biodegradável e não poluente, além de ser um mecanismo de geração de renda e emprego para o homem do campo.

Nesse contexto o interesse em compreender profundamente as características e as propriedades estruturais das fibras naturais utilizadas como reforço nos materiais compósitos, tem crescido, visando adaptar esses materiais para exigências de diferentes funcionalidades dentro das tecnologias de ponta.

Segundo Silva [3], o Brasil é um dos países com uma grande produção e variedade de fibras naturais, tanto as de origem vegetal, quanto animal ou mineral. Diante desta grande variedade, a utilização das fibras vegetais como reforço em compósitos de matriz polimérica é algo que vem sendo estudado com ênfase nas últimas décadas, como em trabalhos desenvolvidos por Esmeraldo [4]; Felipe [5]; Dantas [6], visando o fator econômico, já que as fibras naturais quando comparadas as fibras sintéticas possuem um baixo custo de obtenção, além de serem materiais provenientes de fontes renováveis, atóxicos e possuírem baixa massa específica, utilizam baixo custo de energia durante a sua produção, além de estimular empregos nas áreas rurais.

Dentre as diversas fibras naturais destacam-se na utilização de materiais compósitos as fibras de sisal, curauá, juta, coco etc, as quais apresentam boas propriedades de tração e impacto em diversas matrizes como poliéster, epóxi e fenólicas, afirma Mochnacz [7].

No entanto, as partículas cerosas presentes na superfície da fibra, minimizam a adesão fibra/matriz, fazendo com que sejam diminuídas também algumas propriedades mecânicas do compósito, além disso, possuem características hidrofílicas diminuindo assim o uso de compósitos reforçados com fibras naturais.

Buscando melhorar as propriedades adesivas da fibra/matriz nos compósitos reforçados com fibras naturais, modificações químicas ou físicas da fibra, matriz ou ambos os componentes têm sido realizadas, como por exemplo, em pesquisas realizadas por Angrizani [8]; Sreekumar [9] e Santos [10], que utilizaram hidróxido de sódio e apresentaram mudanças na maioria dos casos positivas . Segundo Cao [11], isto se deve à modificação da estrutura celular da fibra diminuindo seu diâmetro pela dissolução da hemicelulose e a remoção de lignina que são materiais de cimentação.

Desta forma, este trabalho tem como objetivo caracterizar as fibras de sisal e sisal tratado com hidróxido de sódio $(\mathrm{NaOH})$ a $3 \%$, bem como avaliar as propriedades mecânicas de compósitos de matriz polimérica de resina poliéster ortoftálica

\footnotetext{
* Contribuição técnica ao $69^{\circ}$ Congresso Anual da ABM - Internacional e ao 14ํㅡㄹ ENEMET - Encontro Nacional de Estudantes de Engenharia Metalúrgica, de Materiais e de Minas,21 a 25 de julho de 2014, São Paulo, SP, Brasil.
} 


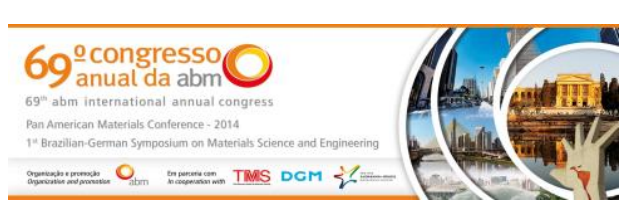

reforçados com tecidos unidirecionais destas fibras, além de, analisar a influência do tratamento químico na superfície da fibra de sisal para o desempenho mecânico do compósito sisal/poliéster.

\section{MATERIAIS E MÉTODOS}

Neste capitulo serão descritos os materiais e métodos utilizados no processo de fabricação dos compósitos bem como os ensaios realizados nos mesmo (densidade e tração) durante o período de realização desta pesquisa.

\subsection{Preparação das Fibras de Sisal e Análise de Suas Propriedades Mecânicas}

As fibras de sisal como podem ser vistas na Figura 2.1 foram recebidas já beneficiadas pela empresa COPISIJU, localizada na cidade de João Câmara, no estado do Rio Grande do Norte.

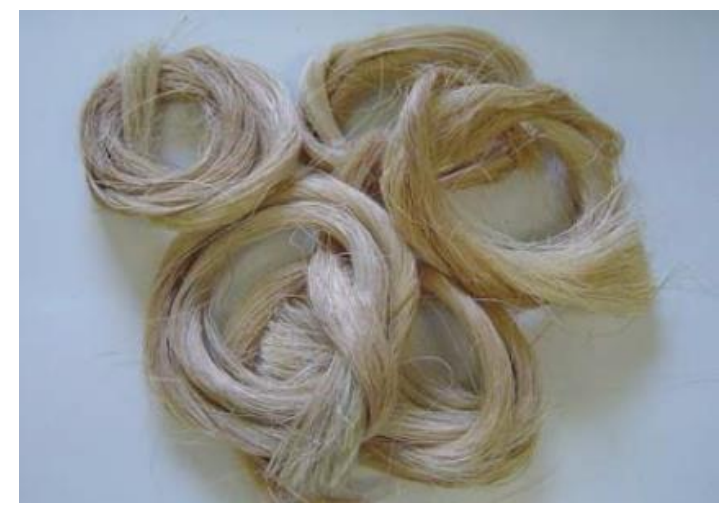

Figura 2.1 - Fibras de sisal como recebidas

O primeiro passo para a análise das propriedades mecânicas das fibras, foi verificar o seu diâmetro. Utilizando o projetor de perfil Mitutoyo PH-A14, foram medidas dez amostras de fibra de sisal, o valor do seu diâmetro foi obtido a partir da média de cinco pontos ao longo do comprimento de cada amostra e determinada a sua média. Após a determinação do diâmetro, foram realizados ensaios de tração nestas amostras. Utilizando o equipamento TENSOLAB AUTOMÁTICO 3000 MESDAN do laboratório de engenharia têxtil da UFRN (Erro! Fonte de referência não encontrada.) foram ensaiadas 10 amostras de cada tipo de fibra com comprimento útil de $100 \mathrm{~mm}$, utilizando velocidade de $10 \mathrm{~mm} / \mathrm{min}$, para se obter as constantes de engenharia destas fibras tais como o módulo de elasticidade e a tensão última de acordo com as equações 3.1 à 3.3, onde $\sigma$ é a tensão aplicada (MPa), $P$ é a carga aplicada durante o ensaio $(\mathrm{N})$, $A$ é a área do fio utilizado, $E$ é o Módulo de elasticidade (GPa), $\varepsilon$ é a deformação de ruptura, $\Delta l$ é a variação de comprimento $(\mathrm{mm})$ do CP e lo é o comprimento inicial do CP. Em todos os casos, foram calculados os valores médios para se obter o desvio padrão das amostras.

$$
\begin{gathered}
\sigma=\frac{P}{A} \\
E=\frac{\sigma}{\varepsilon} \\
\varepsilon=\frac{\Delta l}{l_{o}}
\end{gathered}
$$

\footnotetext{
* Contribuição técnica ao $69^{\circ}$ Congresso Anual da ABM - Internacional e ao 14ํㅡㄹ ENEMET - Encontro Nacional de Estudantes de Engenharia Metalúrgica, de Materiais e de Minas,21 a 25 de julho de 2014, São Paulo, SP, Brasil.
} 


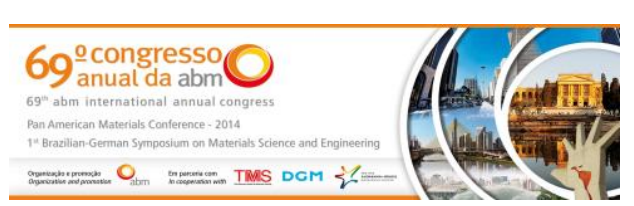

Vale ressaltar que o cálculo do módulo de elasticidade foi realizado mediante análise dos gráficos tensão $x$ deformação de cada corpo de prova, sendo utilizado valores de tensão e deformação ocorridos na região linear dos mesmos.

\subsection{Processo de Fabricação dos Tecidos}

O primeiro passo para confecção dos tecidos foi a preparação das mechas. Para os tecidos de fibras de sisal foram utilizadas mechas com 45 fios e massa de $1,5 \mathrm{~g}$, enquanto que para os tecidos de curauá foram necessários 90 fios por mecha, para se obter esta mesma massa. Após preparação das mechas, estas foram dispostas em um tear com dimensão de 400 x 400 mm, após ser dispostas no urdume, as mechas foram entrelaçadas pela trama composta por mechas de 5 fios, apenas para efeito de sustentação do tecido, conforme se verifica na Figura 3.6.

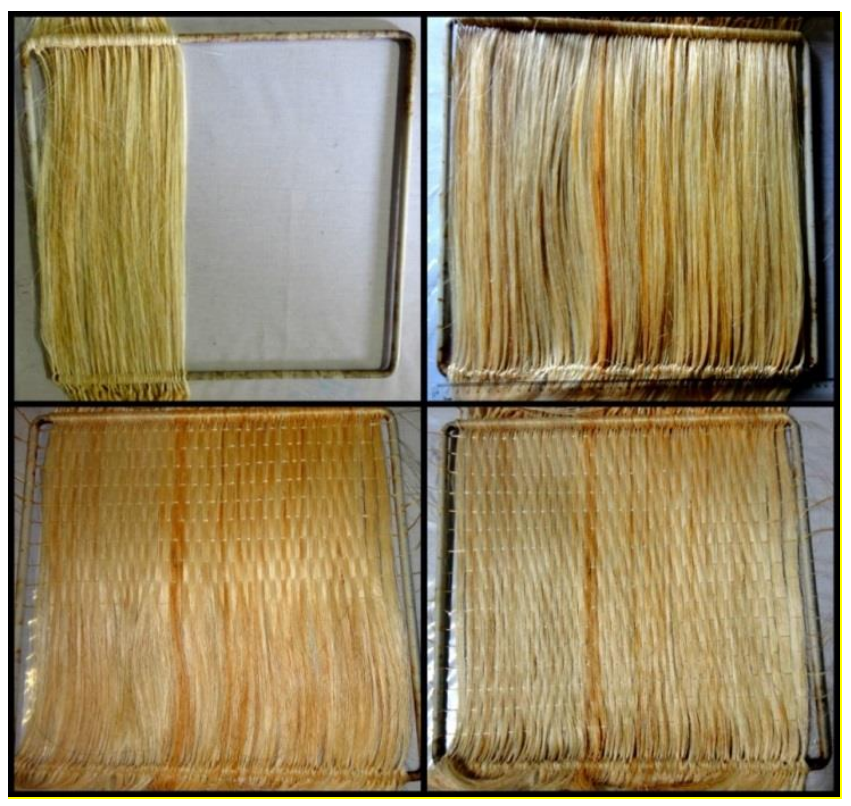

Figura 2.2b - Processo de fabricação do tecido unidirecional

\subsection{Tratamento Superficial}

O método realizado para o tratamento bem como a escolha da porcentagem de Hidróxido de sódio utilizada, foi definido mediante a revisão bibliográfica apresentada no item 2.5. Desta forma, o tratamento nos tecidos de sisal, foi feito com as fibras ainda dispostas no tear, de forma que este foi imerso na solução de hidróxido de sódio $(\mathrm{NaOH})$, a $3 \%$ por $1 \mathrm{~h}$, e após isto o tecido foi submetido à diversos banhos de água destilada afim de se obter um $\mathrm{pH}$ neutro para o mesmo. Após a retirada do hidróxido, mediante a lavagem com água destilada o tecido foi retirado do tear e colocado na estufa à $80^{\circ} \mathrm{C}$, para secar por $24 \mathrm{~h}$.

\subsection{Processo de Fabricação da Lâmina}

O processo de fabricação utilizado foi a laminação manual (hand lay-up), realizado na Tecniplas Indústria e Comércio Ltda. Foi utilizada para fabricação das lâminas, a resina poliéster ortoftálica, e para catalisação se fez uso do peróxido de metil-etil-

\footnotetext{
* Contribuição técnica ao $69^{\circ}$ Congresso Anual da ABM - Internacional e ao 14ํㅡㄹ ENEMET - Encontro Nacional de Estudantes de Engenharia Metalúrgica, de Materiais e de Minas,21 a 25 de julho de 2014, São Paulo, SP, Brasil.
} 


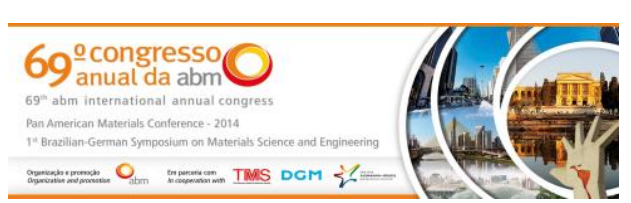

cetona (MEKP) numa proporção de $1 \%$ do volume de resina. O processo de cura ocorreu em temperatura ambiente.

\subsection{Corte dos Corpos de Prova}

O corte de todos os corpos de provas dos ensaios mecânicos foi executado utilizando uma máquina de corte à laser, garantindo assim fidelidade nas dimensões exigidas pelas normas e principalmente precisão no corte do entalhe dos corpos de prova de iosipescu, além de prevenir superaquecimento, entalhes e arranque de fibras que podem ocorrer durante o corte, evitando a interferência desta etapa do processo de fabricação, no desempenho mecânico e na característica física do produto final.

\subsection{Ensaio de Densidade}

Para a realização do teste de densidade os corpos de provas foram cortados mediante a norma ASTM D 792 [12], com dimensão de 25 × $25 \mathrm{~mm}$ para ambos os laminados confeccionados. Sendo a densidade do compósito determinada de acordo com a equação 2.4:

$$
D=\frac{a}{(a+w+b)} x f
$$

Onde $D$ é a densidade do compósito $\left(\mathrm{g} / \mathrm{cm}^{3}\right)$, a é massa da amostra seca $(\mathrm{g}), w$ é a massa do fio parcialmente imerso em água (g), $b$ massa do fio com as amostras imersas em água $(\mathrm{g})$, e $f$ o fator de correção de temperatura.

Para a pesagem das amostras e cálculo da densidade volumétrica utilizou-se uma balança de marca Sartorios com capacidade máxima de $210 \mathrm{~g}$ e precisão $0,0001 \mathrm{~g}$.

\subsection{Ensaio de Tração Uniaxial dos Compósitos Fabricados}

A realização deste ensaio foi mediante a norma ASTM D3039 [13], utilizando corpos de prova com dimensões de $15 \mathrm{~mm}$ de largura, $127 \mathrm{~mm}$ de distância entre os galgos, $250 \mathrm{~mm}$ de comprimento total e $3,9 \mathrm{~mm}$ de espessura média.

\section{RESULTADOS E DISCUSSÃO}

A seguir, serão apresentados os resultados obtidos com a caracterização mecânica das fibras de sisal e sisal tradado quanto à tração, bem como os resultados de ensaio de densidade volumétrica para os compósitos reforçados por estas fibras, além do comportamento mecânico destas lâminas quando submetidas a ensaios de tração uniaxial, e ainda, análise da fratura.

\subsection{Ensaio de densidade volumétrica}

$\mathrm{Na}$.

Tabela1, podem ser observadas as densidades das lâminas e seus respectivos desvios padrões.

Tabela 3.1 - Resultados dos ensaios de densidade das lâminas

\footnotetext{
* Contribuição técnica ao $69^{\circ}$ Congresso Anual da ABM - Internacional e ao 14ํㅡㄹ ENEMET - Encontro Nacional de Estudantes de Engenharia Metalúrgica, de Materiais e de Minas,21 a 25 de julho de 2014, São Paulo, SP, Brasil.
} 

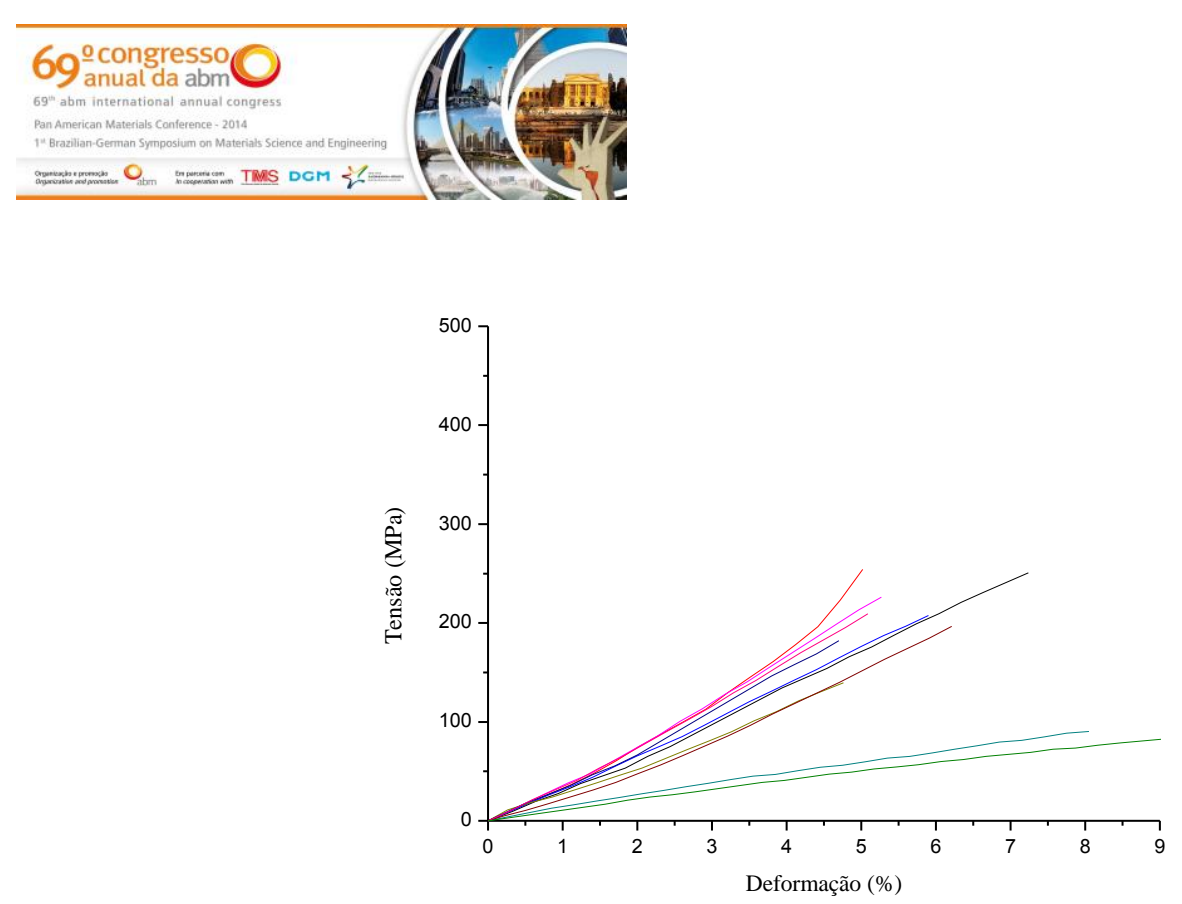

Figura 3.2 - Curva tensão $x$ deformação da fibra de sisal tratado

Comparando-se as curvas das Figura 3.1 efigura 3.2, percebe-se uma maior dispersão, bem como um comportamento bastante diferenciado da fibra de sisal não tratado, à medida que ocorre diversas quedas de tensão. Este fato pode ser explicado pela estrutura da fibra de sisal, que se assemelha a um material compósito, pois é constituída essencialmente por microfibrilas de celulose, comportando-se como o reforço, envoltos por hemicelulose, lignina e outras partículas cerosas em pequenas quantidades, que juntas assumem o papel de matriz. Assim, ao serem tracionadas, estas fibrilas se rompem gradativamente até a ruptura total da fibra, causando o comportamento visto na Figura 3.1. É possível também observar que ao se retirar a camada cerosa da fibra (Figura 3.3.2), tem-se como consequência uma menor dispersão, visto que as fibrilas estão parcialmente sem a proteção das partículas cerosas, fragilizando-as e fazendo com que elas se rompam de forma mais frágil.

Para uma melhor comparação dos resultados, desenhou-se os gráficos de barras das figuras 3.3 e 3.4 para a tensão última das fibras e o seu módulo, respectivamente.

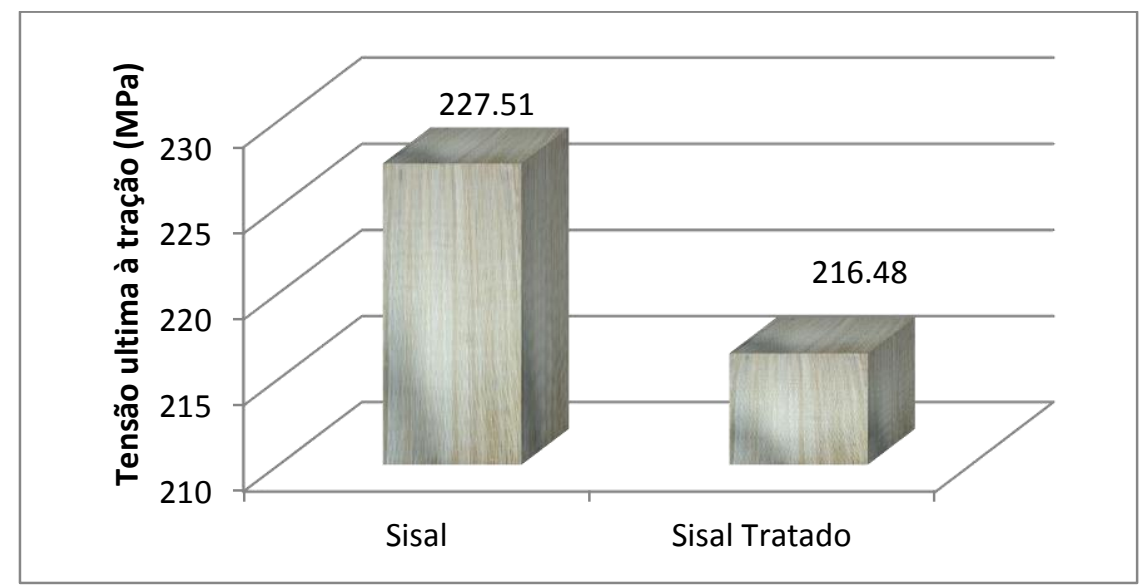

Figura 3.3 - Tensão última à tração das fibras

Percebe-se na figura 3.3 maior resistência à tração das fibras de sisal não tratado, sendo essa diferença de $17 \%$, comprovando que o tratamento químico superficial na

\footnotetext{
* Contribuição técnica ao $69^{\circ}$ Congresso Anual da ABM - Internacional e ao 14ํㅡㄹ ENEMET - Encontro Nacional de Estudantes de Engenharia Metalúrgica, de Materiais e de Minas,21 a 25 de julho de 2014, São Paulo, SP, Brasil.
} 


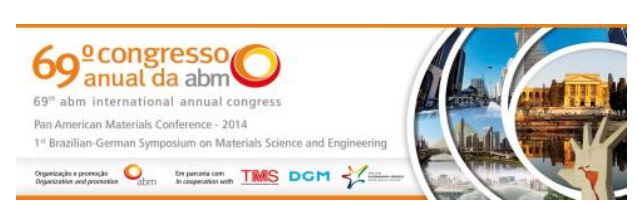

fibra de sisal fragiliza de forma expressiva esta fibra, além disso, o tratamento também diminuiu em $69 \%$ a rigidez da fibra, como mostrado na figura 3.4.

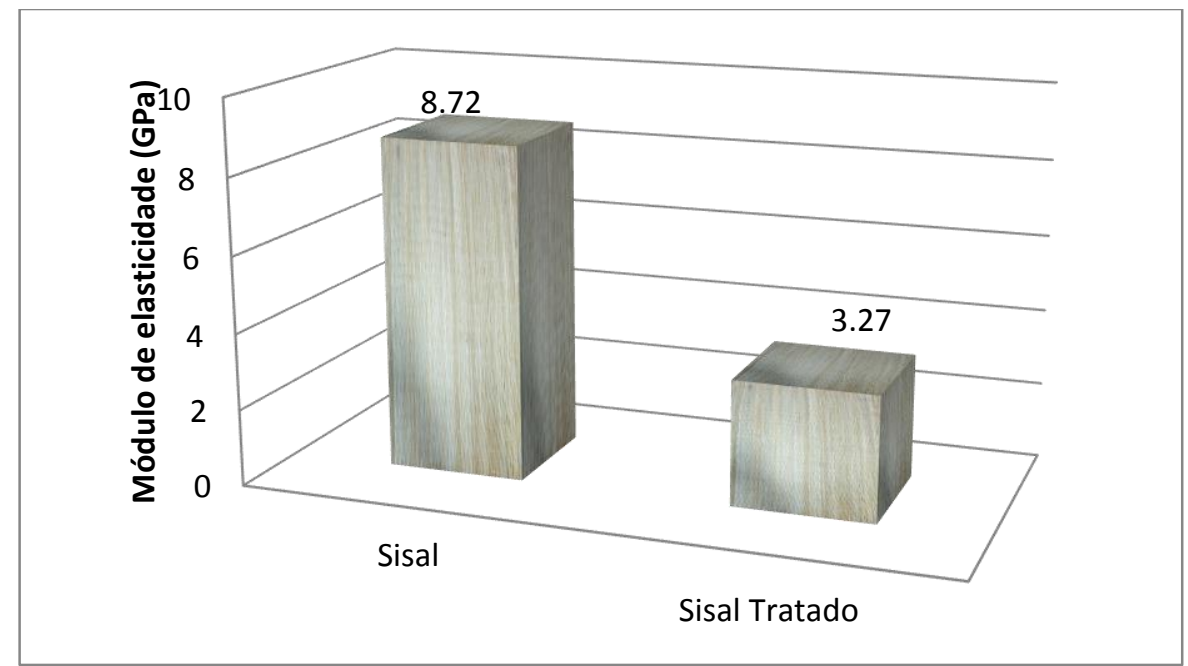

Figura 3.4 - Módulo de elasticidade à tração das fibras

\subsection{Ensaio de Tração Uniaxial nas Lâminas}

Neste item serão apresentados os resultados dos ensaios mecânicos de tração uniaxial realizados nos compósitos poliméricos reforçados por tecido unidirecional de sisal.

\subsubsection{Lâminas reforçadas com tecido unidirecional}

Apresentam-se nas Erro! Fonte de referência não encontrada.5 e 3.6 os diagramas Tensão x Deformação das lâminas reforçadas por sisal e sisal tratado, ensaiados a tração, na qual se percebe que todas as lâminas possuem grande dispersão, fato este já esperado, pois é uma característica intrínseca à utilização da fibra natural como reforço em compósitos poliméricos.

Outro ponto importante percebido nestas figuras é que após um determinado valor de tensão (aproximadamente 20MPa), ocorreu para todos os materiais sucessivas quedas de tensão ocasionadas por pequenas trincas na matriz da lâmina. Este fenômeno ocorre principalmente em lâminas (1 camada) Silva [15], em laminados (mais de uma camadas) este fenômeno é mais difícil ser percebido, já que, caso ocorra a fratura na matriz esta deveria iniciar um processo de delaminação entre camadas. Apesar de ser mais difícil de apresentar em laminados, foi verificado no trabalho de Silva [3] que um laminado de 5 camadas de tecido de sisal apresentou este comportamento.

\footnotetext{
* Contribuição técnica ao 69ำ Congresso Anual da ABM - Internacional e ao 14ํㅡㄹ ENEMET - Encontro Nacional de Estudantes de Engenharia Metalúrgica, de Materiais e de Minas,21 a 25 de julho de 2014, São Paulo, SP, Brasil.
} 


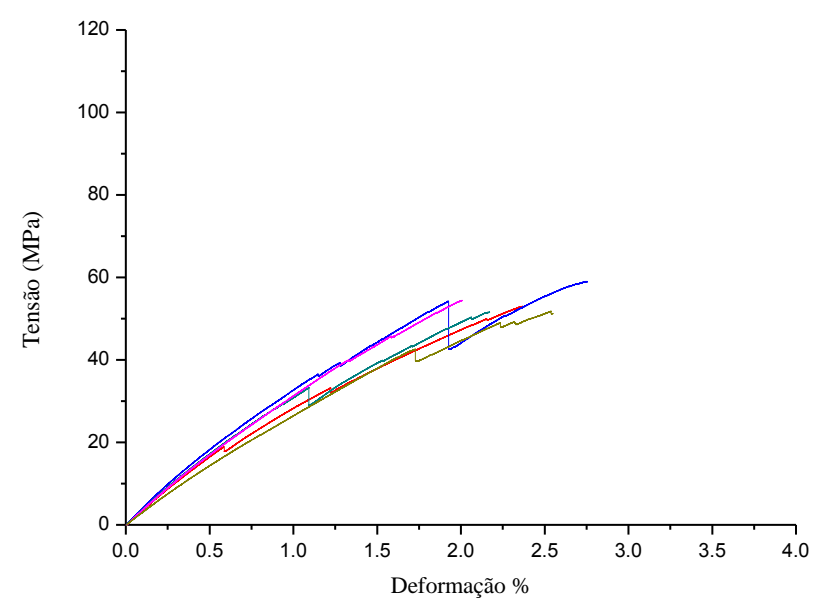

Figura 3.5 - Curva Tensão x Deformação da lâmina de sisal unidirecional

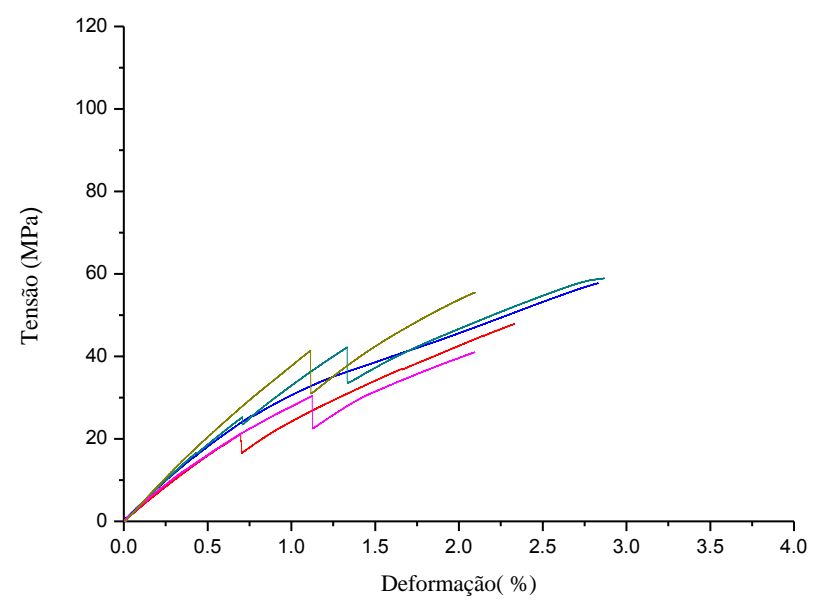

Figura 3.6 - Curva Tensão x Deformação da lâmina de sisal tratado unidirecional

Analisando a Figura 3.7, percebe-se que, contrário ao que aconteceu no ensaio de tração da fibra tratada, o compósito que utilizou esta fibra como reforço, obteve maior tensão de ruptura que o compósito de fibra não tratada. O aumento foi de 2,15 $\mathrm{MPa}$ (diferença percentual de 4,13\%). Confirmando assim a literatura, em que Rong [16] e Sreekumar [9] também aumentaram a resistência à tração com tratamento superficial na fibra de sisal.

* Contribuição técnica ao $69^{\circ}$ Congresso Anual da ABM - Internacional e ao 14ํㅡㄹ ENEMET - Encontro Nacional de Estudantes de Engenharia Metalúrgica, de Materiais e de Minas,21 a 25 de julho de 2014, São Paulo, SP, Brasil. 

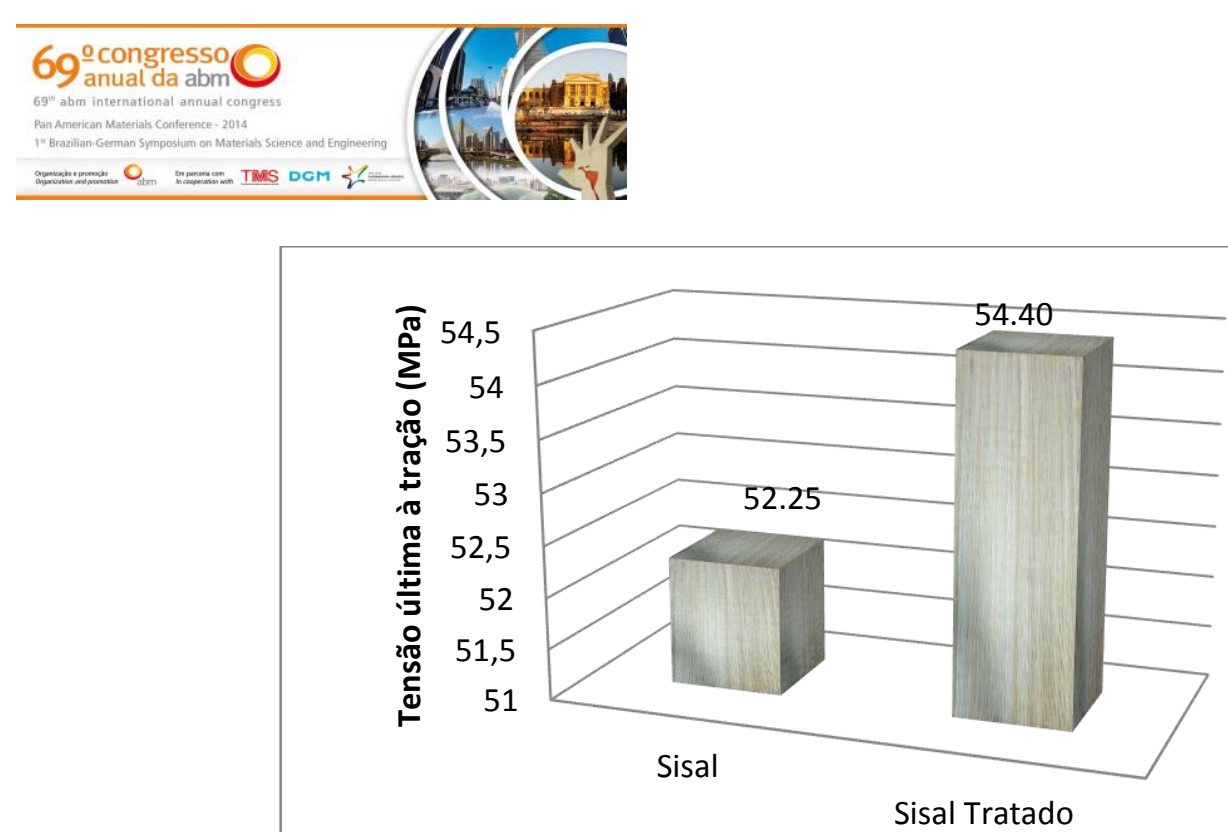

Figura 3.7 - Tensão última à tração das lâminas com reforço unidirecional disposto à $0^{\circ}$

Em relação ao módulo de elasticidade, o compósito que utilizou a fibra tratada obteve melhor desempenho quando comparado ao de reforço não tratado, como pode ser visualizado no gráfico abaixo (figura 3.8), aumentando em 10,8\% a rigidez da lâmina e $21,2 \%$ a deformação. Confirmando assim, a maior interação do conjunto fibra/matriz quando retirada a camada cerosa da superfície da fibra de sisal.

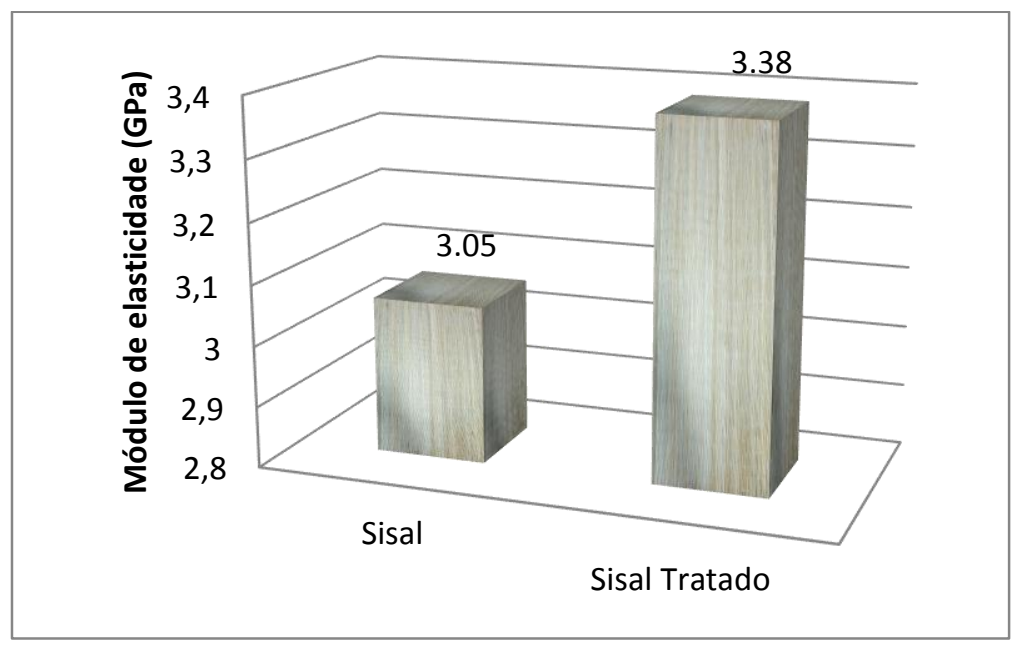

Figura 3.8 - Módulo de elasticidade à tração das lâminas com reforço unidirecional disposto à $0^{\circ}$

\subsubsection{Análise da macroscópica da fratura}

Foram analisadas macroscopicamente as fraturas dos compósitos ensaiados à tração e posteriormente classificados quanto ao modo de falha aceitável pela norma ASTM D3039.

As Figuras 3.9 e 3.10, mostram as fraturas obtidas no ensaio de tração dos compósitos reforçados com fibras de sisal e sisal tratado. Em todos os casos, caracterizam-se fraturas aceitas pela norma ASTM 3039.

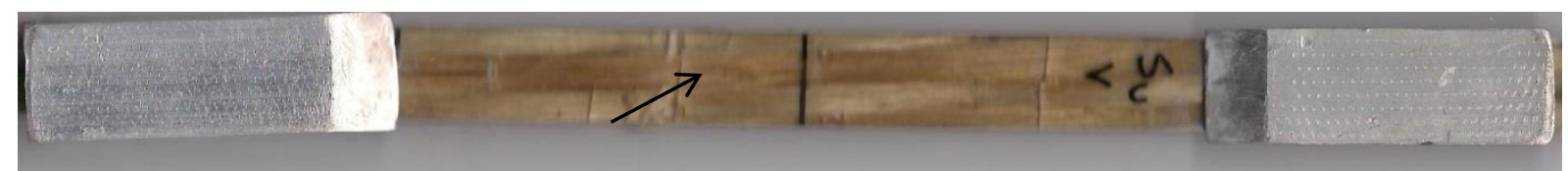

Figura 3.9 - Fratura do compósito reforçado com tecido de sisal

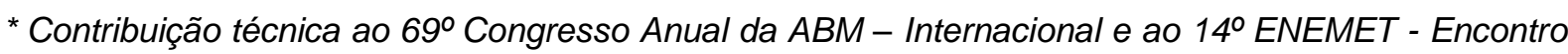
Nacional de Estudantes de Engenharia Metalúrgica, de Materiais e de Minas,21 a 25 de julho de 2014, São Paulo, SP, Brasil.
} 

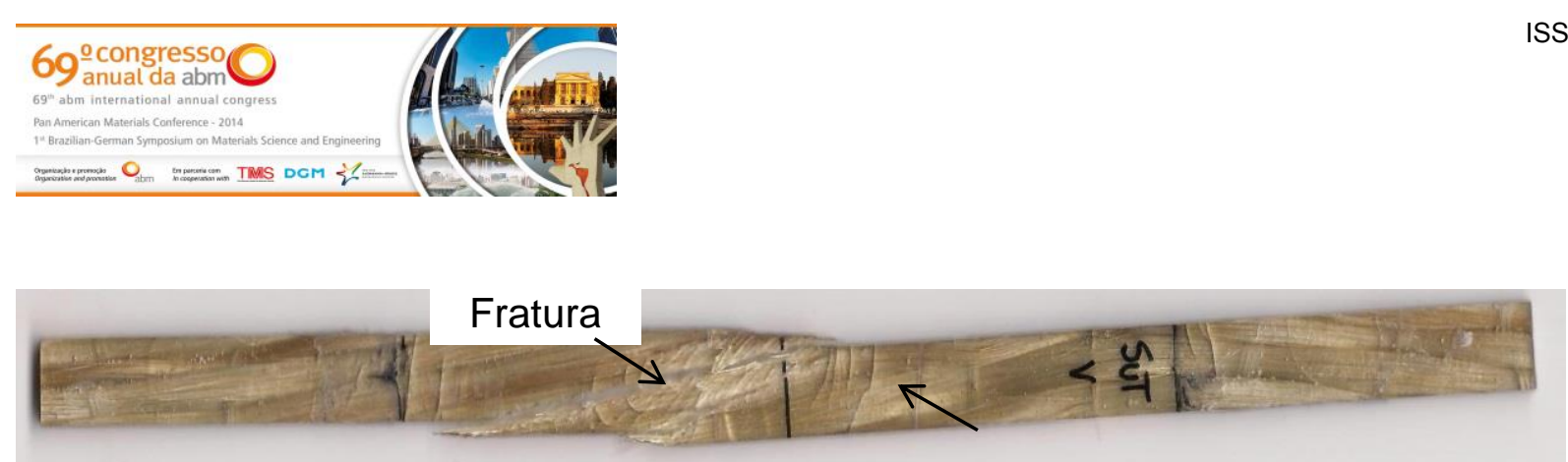

Figura 3.10 - Fratura do compósito reforçado com tecido de sisal tratado

\section{CONCLUSÃo}

De acordo com os resultados apresentados, pode-se observar que o tratamento químico superficial com hidróxido de sódio na fibra de sisal fragiliza de forma expressiva esta fibra e quando utilizada como reforço no compósito polimérico, aumentou a densidade do compósito, a tensão de ruptura em 4,3\%, bem como 0 módulo de elasticidade em $10,8 \%$. Confirmando dessa forma a literatura no que diz respeito ao aumento da resistência mecânica em compósitos reforçados com fibras vegetais de superfície tratada quimicamente, que afirma de um modo geral que a medida em que o Hidróxido de sódio $(\mathrm{NaOH})$ reage com grupos hidroxilas da hemicelulose do material ocorre a destruição da estrutura celular e, assim, as fibras são divididas em filamentos, fenômeno este denominado como fibrilação, que rompe feixe de fibras tratadas diminuindo seu diâmetro pela dissolução da hemicelulose e a remoção de lignina que são materiais de cimentação acarretando num aumento da área superficial efetiva disponível para contato com a matriz, o que leva a um melhor acondicionamento das cadeias de celulose, afetando na maioria dos casos positivamente as características mecânicas das fibras

\section{Agradecimentos}

Os autores agradecem ao CNPq pelo apoio financeiro.

\section{REFERÊNCIAS}

1 Santos JKD. Influência da configuração do tecido e do tratamento superficial das fibras nas propriedades mecânicas de compósitos reforçados com fibras naturais.

Dissertação de mestrado. Natal: Universidade Federal do Rio Grande do Norte; 2013

2 Gay D.- Materiaux Composites. Paris: Editions Hermès, 1991.

3 Silva CC. - Influência da umidade nas propriedades mecânicas dos compósitos poliméricos híbridos (sisal/ vidro). Dissertação de mestrado - UFRN, Natal 2012.

4 Esmeraldo MA. - Preparação de novos compósitos suportados em matriz de fibra vegetal/natural. Dissertação de mestrado - UFC, Fortaleza 2006.

5 Felipe RCTS. - Envelhecimento ambiental acelerado em PRF a base de tecidos híbridos kevlar/vidro: propriedades e instabilidade estrutural. Tese de doutorado UFRN, Natal, 2012.

6 Dantas CM. - Influência da quantidade de absorção de água nas propriedades mecânicas de compósitos poliméricos de manta de fibra de vidro e tecido juta Dissertação de Mestrado - UFRN, 2011.

7 Mochnacz S, Amico SC, Sydenstricker THD. Caracterização e modificação Superficial de Fibras de Sisal Para Utilização em Compósitos Poliméricos.- Congresso em Ciências de Materiais do Mercosul, Joinville-SC, Brasil. 2002.

8 Angrizani CA, Vieira CAB, Zattera AJ; Freire E, Santana RMC. Amico, S.C. Influência do comprimento da fibra de sisal e do seu tratamento químico nas propriedades de compósitos com poliéster. In $17^{\circ}$ Congresso brasileiro de engenharia e ciência dos materiais, Foz do Iguaçu-PR, Brasil. 2006.

\footnotetext{
* Contribuição técnica ao $69^{\circ}$ Congresso Anual da ABM - Internacional e ao 14ํㅡㄹ ENEMET - Encontro Nacional de Estudantes de Engenharia Metalúrgica, de Materiais e de Minas,21 a 25 de julho de 2014, São Paulo, SP, Brasil.
} 


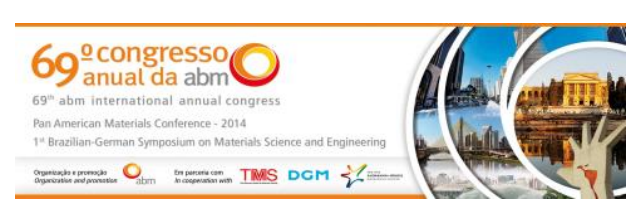

9 Sreekumar, P.A., Thomas S.P., Saiter, J.M., Kuruvilla, J., Unnikrishnan, G., Sabu, T. "Effect of fiber surface modification on the mechanical and water absorption characteristics of sisal/polyester composites fabricated by resin transfer molding". Elsevier Science Ltda; August 2009; vol 40: pp 1777-1784.

10 Santos, J. K. D. - Busca da melhoria do desempenho mecânico do ecomaterial sisal/poliéster. TCC - IFRN. Natal, 2011.

11 Cao Y, Shibata S, Fukumoto I. Mechanical properties of biodegradable composites reinforced with bagasse fiber before and after alkali treatments. Compos Part A: Appl Sci Manuf, 2006; 37:423-9.

12 American society for testing and materials. ASTM D792-08. Standard test methods for specific gravity and density of plastics by displacement. [S.I.]: ASTM. 1944.

13 American society for testing and materials. ASTM D3039-08. Standard test methods for tensile properties of polymer matrix composites. Philadelphia: [s.n.]. 1971.

14 Martin AR. Caracterização química e estrutural de fibra de sisal da variedade agave sisalana. Polímeros: Ciência e Tecnologia, 2009; 19(1): 40-46.

15 Silva CC. Fabricação de tecido de sisal: Elemento de reforço em matriz polimérica. Monografia - Instituto Federal de Educação, Ciência e Tecnologia do Rio Grande do Norte, Natal, 2010.

16 Rong MZ, Zhang MQ, Liu Y, Yang GC, Zeng HM. The effect of fiber treatment on the mechanical properties of unidirectional sisal-reinforced epoxy composites. - Elsevier Science Ltda; March 2001; vol 61: pp 1437-1447.

* Contribuição técnica ao $69^{\circ}$ Congresso Anual da ABM - Internacional e ao 14ํㅡㄹ ENEMET - Encontro Nacional de Estudantes de Engenharia Metalúrgica, de Materiais e de Minas,21 a 25 de julho de 2014, São Paulo, SP, Brasil. 